\title{
Adherence and persistence with branded antidepressants and generic SSRIs among managed care patients with major depressive disorder
}

\author{
This article was published in the following Dove Press journal: \\ ClinicoEconomics and Outcomes Research \\ 14 March 2011 \\ Number of times this article has been viewed
}

\author{
Xianchen Liu' ${ }^{1,2}$ \\ Yi Chen ${ }^{3}$ \\ Douglas E Faries ${ }^{3}$ \\ 'Former employee, Eli Lilly and \\ Company, Indianapolis, Indiana, USA; \\ ${ }^{2}$ Indiana University Department of \\ Psychiatry, Indianapolis, Indiana, USA; \\ ${ }^{3}$ Eli Lilly and Company, Indianapolis, \\ Indiana, USA
}

\begin{abstract}
Objective: This study compared adherence and persistence of three branded antidepressants: the serotonin and norepinephrine reuptake inhibitors (SNRIs) duloxetine and venlafaxine XR, and the selective serotonin reuptake inhibitor (SSRI) escitalopram; and generic selective SSRIs, and examined demographic and clinical predictors of adherence and persistence in patients with major depressive disorder in usual care settings.
\end{abstract}

Method: A total of 44,026 patients (18 to 64 years) from a large commercial administrative claims database were classified as initiators of duloxetine $(n=7,567)$, venlafaxine XR $(n=6,106)$, escitalopram ( $n=10,239)$, or generic SSRIs $(n=20,114)$ during 2006. Adherence was defined as the medication possession ratio of $\geq 0.8$ and persistence as the length of therapy without exceeding a 15-day gap. Pairwise comparisons from multivariate logistic regression and Cox proportional hazards models were performed to examine predictors of adherence and persistence.

Results: Adherence rate after one year was significantly higher in duloxetine recipients $(38.1 \%)$ than patients treated with venlafaxine XR (34.0\%), escitalopram (25.4\%), or generic SSRIs (25.5\%) (all $P<0.01)$. Duloxetine recipients stayed on medication longer (158.5 days) than those receiving venlafaxine XR (149.6 days), escitalopram (129.1 days), or generic SSRIs (130.2 days) (all $P<0.001)$. Compared with patients treated with escitalopram or generic SSRIs, venlafaxine $\mathrm{XR}$ recipients had better adherence and longer persistence $(P<0.001)$. In addition, being aged 36 years or more, hypersomnia, anxiety disorders, and prior use of antidepressants were associated with increased adherence and persistence, while the opposite was true for comorbid chronic pain conditions, alcohol and drug dependence, and prior use of amphetamine.

Conclusion: Compared with SSRIs, the SNRIs appear to have better adherence and persistence. Among SNRIs, duloxetine had statistically significantly better adherence and persistence than venlafaxine XR, though differences were relatively small and further research is needed to assess whether these translate into clinically and economically meaningful outcomes. Adherence and persistence with antidepressant therapy were associated with age, multiple comorbid conditions, and prior use of medications.

Keywords: treatment adherence, length of therapy, antidepressants, major depression, retrospective analysis

\section{Introduction}

Major depressive disorder (MDD) is among the most prevalent and costly psychiatric disorders. ${ }^{1-3}$ Antidepressants are the mainstay treatment of MDD and optimal antidepressant treatment includes the selection of the right medication and delivery at a fully therapeutic dose for a sufficient treatment duration. ${ }^{4,5}$ 
However, antidepressant nonadherence remains a challenging problem in the treatment of depression. Treatment is often accompanied by premature discontinuation and switching of antidepressant medications, ${ }^{8}$ which may be associated with likelihood of relapse and higher healthcare costs. ${ }^{9} 10$ Research demonstrates that $28 \%$ of patients discontinue use of their antidepressant medication within the first month, and by three months, at least $40 \%$ have discontinued use. ${ }^{11}$ Approximately $16 \%$ of patients remain on therapy for more than 90 days without evidence of therapy changes, titration in dose, or being only partially compliant. ${ }^{12}$ The early discontinuation of antidepressant medication is statistically associated with a $77 \%$ increase in the risk of relapse. ${ }^{10}$

In many cases, poor adherence or discontinuation of therapy was thought to be an effect related primarily to adverse events or lack of therapeutic response. ${ }^{13}$ However, there are multiple factors that may put patients at risk for poor adherence or discontinuation. Many patient-related, diseaserelated, medication-related, and physician-related factors, along with the health care system and costs may influence a patient's adherence to medication therapy. ${ }^{13-21}$

The second generation of antidepressants, such as selective serotonin (5-HT) reuptake inhibitors (SSRIs) and serotonin and norepinephrine (NE) reuptake inhibitors (SNRIs), are commonly used in the treatment of depression. Although findings are inconsistent, some studies suggest that SNRIs that modulate both 5-HT and NE activity may be more effective than SSRIs for patients with more severe major depression. ${ }^{22,23}$ Although some antidepressants are grouped into the same class, they may have different pharmacological and clinical profiles. ${ }^{24-27}$

At the time of the analyses, three branded antidepressants commonly used in the treatment of depression in the United States were two SNRIs: duloxetine (DLX) and venlafaxine (VLX) and one SSRI: escitalopram (ECP). Recent research showed that DLX-treated patients had more comorbid conditions, especially chronic pain-related diseases, and that ECP-treated patients were very similar to generic SSRI (GSSRI)-treated patients in demographic characteristics and comorbid conditions..$^{28}$ Branded antidepressants are usually more expensive than GSSRIs. However, little is known about treatment patterns and the clinical and economic consequences of patients treated with branded antidepressants compared with GSSRIs. The objectives of this study were to examine treatment adherence and persistence with the three branded antidepressants DLX, VLX, and ECP, and GSSRIs in the treatment of major depression, and to examine demographical and clinical predictors of adherence and persistence with antidepressant therapy in the usual clinical setting.

\section{Patients and Methods Data source and sample selection}

This retrospective analysis of claims data employed medical, pharmacy, and enrollment information from the Thomson Reuters MarketScan Commercial Claims and Encounters Databases, which includes de-identified administrative claims databases of employees, spouses, and dependents with employer-sponsored commercial insurance. Inpatient and outpatient medical and pharmacy claims are linked to enrollment data for a variety of plan types. This database captures the annual health care information of approximately 20 million individuals, including inpatient and outpatient medical claims linked to pharmacy and enrollment data for a variety of health insurance plan types. Each medical claim includes at least one International Classification of Diseases (ICD), 9th Edition, Clinical Modification (ICD-9-CM) diagnostic code to describe the patient's clinical condition. All pertinent patient information in the database was encrypted and deidentified. The MarketScan database is publicly available as a fee-for-service database and has been used for a number of previous retrospective and prospective analysis projects. ${ }^{29,30}$

Patients were included in the study if they had a first prescription fill for DLX, VLX, ECP, or a GSSRI in 2006, had no active prescriptions of the same study medication in the three months prior to the index date, and had one or more inpatient or outpatient claims associated with a diagnosis of MDD (ICD-9-CM: 296.2 and 296.3) one year prior to or one month after the study medication was initiated. Patients had to be 18 to 64 years of age, commercially insured, and have continuous enrollment in the previous and following twelve months. Patients initiated on more than one study medication (DLX, VLX, ECP, or GSSRIs) during 2006 at different times were grouped into the first medication initiation cohort while those starting on more than one type of study medication on the index date were excluded from the study. Patients were grouped into four mutually exclusive cohorts: DLX, VLX, ECP, or GSSRI. The study design and sample selection are illustrated in Figure 1.

\section{Adherence and persistence}

Medication adherence was assessed using the medication possession ratio (MPR), defined as the sum of the days' supply of study medication within one year after the index date divided by 365 days ${ }^{6,7}$ Adherence to therapy was defined as an MPR $\geq 0.8$. Medication persistence was defined as 


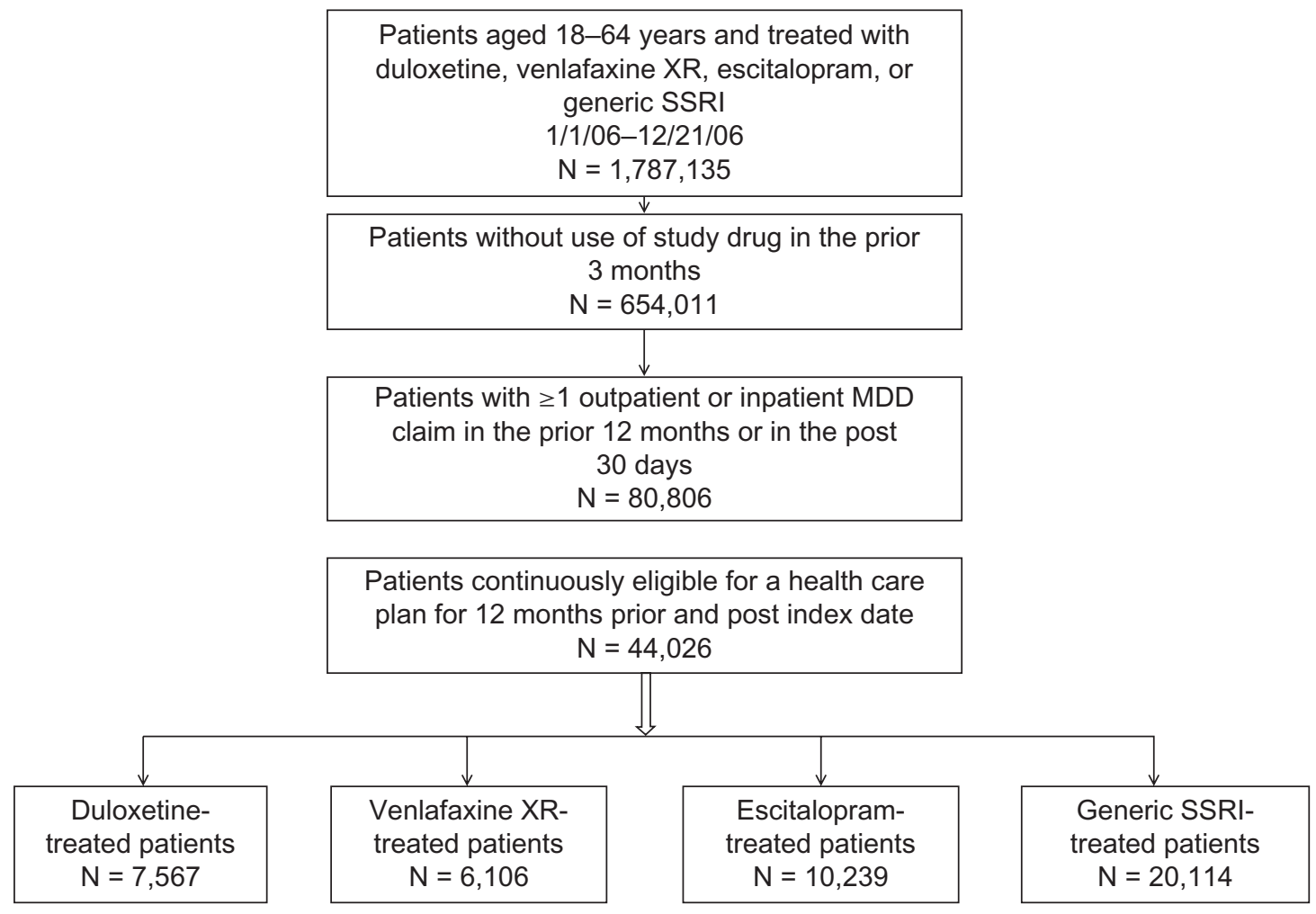

Figure I Summary of study design and sample selection.

Abbreviations: N, number of patients; MDD, major depressive disorder; SSRI, selective serotonin reuptake inhibitor.

the number of days from the index date to the earliest of the ending date of the last prescription, the date of the first gap of more than 15 days between prescriptions, or the end of the study period of twelve months, whichever came first. ${ }^{6,7}$

\section{Pretreatment variables}

Pretreatment variables included demographic characteristics (age, gender, geographic region of residence, and health plan type), comorbidities, and prior use of medications. Comorbidities included 20 chronic pain conditions, sleep disorders, psychiatric disorders, and other physical disorders (see Table 1 for detail) that are associated with safety, efficacy, or adherence of antidepressant treatment. ${ }^{28,31-37}$ Prior use of medications included specified therapeutic classes, as well as selected individual drugs known to be used in treating depression, psychosis, bipolar disorders, anxiety, sleep disorders, or chronic pain that are known to be associated with physicians' choice of antidepressants. ${ }^{4,33,38,39}$ All medications were identified on the basis of National Drug Codes.

\section{Statistical analyses}

Summary statistics were presented as means and standard deviations for continuous variables and as percentages for categorical variables. Demographic characteristics at the index date of study medication initiation, comorbid conditions, and medications used in the preindex period (one year prior to index date) were compared between the four medication cohorts. Chi-square tests were performed for comparisons of categorical variables and analysis of variance for comparisons of continuous variables. Kaplan-Meier survival curves were produced to show times to discontinuation of the four study medication cohorts.

Pairwise comparisons were conducted to examine the differences in adherence and persistence between the four study medication cohorts, adjusting for demographic variables, comorbid conditions, and prior medication use. The Tukey-Kramer method was used to adjust for multiple comparisons.

A multivariate stepwise logistic regression and Cox proportional hazards regression analyses were conducted to determine variables that independently predicted adherence and time to discontinuation (persistence) in the study, adjusting for all the variables in the model. Independent variables for the multivariate stepwise logistic regression included study medications, demographics, comorbid disorders, and concomitant medications in the pre-study period. Odds ratios 
Table I Comorbid conditions and ICD-9-CM diagnosis codes

\begin{tabular}{|c|c|}
\hline & Diagnosis code(s) \\
\hline \multicolumn{2}{|l|}{ Pain conditions } \\
\hline Headaches & 307.8I, 346.xx, 784.0x \\
\hline Rheumatoid arthritis & $7 \mid 4 . x x$ \\
\hline Osteoarthritis & $715 . x x$ \\
\hline \multicolumn{2}{|l|}{ (Osteoarthrosis and } \\
\hline \multicolumn{2}{|l|}{ allied disorders) } \\
\hline \multirow[t]{3}{*}{ Low back pain } & $721.3 x, 722.10,722.32,722.52,722.93,724.02$ \\
\hline & $724.2 x-724.7 x, 738.5 x, 739.3 x, 739.4 x$ \\
\hline & $846,847.2 x$ \\
\hline Fibromyalgia & $729.1 x$ \\
\hline Neuropathic pain & $250.6 x, 357.2 x, 350.1 x, 353.6 x, 053.1 x$ \\
\hline \multicolumn{2}{|l|}{ Psychiatric and } \\
\hline \multicolumn{2}{|l|}{ sleep disorders } \\
\hline Dysthymic disorder & $300.4 x$ \\
\hline Anxiety disorders & $300.0 x, 300.23,308 . x x$ \\
\hline $\begin{array}{l}\text { Alcohol abuse/ } \\
\text { dependence }\end{array}$ & $305.0 x, 303 . x x$ \\
\hline Drug dependence & $304 . x x$ \\
\hline Bipolar disorders & 296.0, 296.I, 296.4-7, 296.80, 296.8I, 296.89 \\
\hline Organic psychosis & 290.xx-294.xx \\
\hline Insomnia & $780.51,780.52$ \\
\hline Hypersomnia & $780.53,780.54$ \\
\hline Obstructive sleep apnea & 327.23 \\
\hline \multicolumn{2}{|l|}{ Other diseases } \\
\hline Diabetes mellitus & 250.xx (except 250.6) \\
\hline Hypertensive diseases & $401 . x x-405 . x x$ \\
\hline Hepatic diseases & $570 . x x-573 . x x$ \\
\hline Renal diseases & $\begin{array}{l}\text { 580.xx-591.xx, 592.0x, 593.0x-593.2x, } \\
593.81,593.9 x\end{array}$ \\
\hline
\end{tabular}

Abbreviations: ICD-9-CM, International Classification of Diseases, 9th Edition, Clinical Modification; MDD, major depressive disorder.

(ORs) and 95\% confidence intervals (CI) from the logistic regression model were used to examine the associations of predicting variables with adherence. The larger the odds ratio was, the higher the likelihood of adherence to the study medication. Hazard ratios (HRs) and 95\% confidence intervals from the Cox proportional hazards model were used to examine associations of predicting variables with time to discontinuation of medication. The smaller the hazard ratio was, the less the likelihood of discontinuation of the medication.

Sensitivity analyses were conducted to compare group differences in adherence and persistence. First, adherence and persistence in the six months after initiation were computed. Second, persistence was estimated using an alternative allowable 30-day gap. Third, 17-ICD classes of systemic diseases as comorbid conditions were used instead of selected diseases for multivariate logistic and Cox proportional hazards regression modeling. All statistical analyses were performed using SAS (v 9.1; SAS institute, Inc, Cary, NC).

\section{Results}

A total of 44,026 patients with continuous enrollment in the health plan for twelve months prior to and post index date were included in the study. Demographic characteristics of the sample by medications are reported in Table 2 . The sample was primarily female and had a mean age ranging from 43.7 to 47.2 years by medications $(P<0.0001)$. Within each medication cohort, approximately one-third of the sample was aged 46 to 55 years. Most of the sample had a Preferred Provider Organization (PPO) health plan which was highest in the DLX group $(P<0.0001)$, and more patients from each drug group lived in the South $(P<0.0001)$.

Figure 2 presents adherence to antidepressant therapy across four medications. In the six months after medication initiation, antidepressant adherence was highest for DLX (48.7\%), followed by VLX (45.9\%), and ECP and GSSRIs $(37.2 \%)$. In the twelve months after medication initiation, adherence rate declined for all medication cohorts. DLX was still highest (38.1\%) and ECP and GSSRIs were lowest (25.4\% and $25.5 \%$, respectively). Pairwise comparisons showed that DLX had a significantly higher adherence rate than the three comparators and that VLX had a significantly higher adherence rate than ECP and GSSRIs at six months and twelve months (all $P<0.01$ ).

In the twelve months after medication initiation, average length of therapy (persistence) was 158.5 days $(\mathrm{SD}=133.9$, median $=95.0)$ with DLX; 149.6 days $(\mathrm{SD}=129.9$, median $=90.0)$ with VLX; 129.1 days $(\mathrm{SD}=119.8$, median $=90)$ with ECP; and 130.2 days $(\mathrm{SD}=120.7$, median $=90.0$ ) with GSSRIs, respectively. Pairwise comparisons showed that the average persistence duration was significantly longer in DLX-treated patients compared to patients treated with VLX $(P<0.001)$, ECP $(P<0.001)$, or GSSRIs $(P<0.001)$. Length of therapy was significantly longer in VLX-treated patients compared to patients treated with ECP $(P<0.001)$ or GSSRIs $(P<0.001)$. However, ECP and GSSRIs did not significantly differ in length of therapy $(P>0.05)$.

Figure 3 shows the Kaplan-Meier curves (time to discontinuation) of different antidepressant therapies in the twelve months after medication initiation. In the first 30 days, about $30 \%$ of patients discontinued their therapy across all treatment groups. After 30 days, DLX and VLX appeared to be different from ECP and GSSRIs and after 90 days, DLX was less likely to be discontinued than VLX. The median time to discontinuation was 95 days in DLX-treated patients, 90 days 
Table 2 Demographic characteristics of patients initiated on duloxetine, venlafaxine XR, escitalopram, or generic SSRIs

\begin{tabular}{|c|c|c|c|c|c|}
\hline & $\begin{array}{l}\text { Duloxetine } \\
(n=7,567)\end{array}$ & $\begin{array}{l}\text { Venlafaxine XR } \\
(n=6,106)\end{array}$ & $\begin{array}{l}\text { Escitalopram } \\
(n=10,239)\end{array}$ & $\begin{array}{l}\text { Generic SSRIs } \\
(n=20,1 \mid 4)\end{array}$ & $P$-values \\
\hline Female gender (\%) & 75.1 & 71.5 & 69.7 & 70.5 & $<0.0001$ \\
\hline \multicolumn{6}{|c|}{ Age group (years) (\%) } \\
\hline $18-25$ & 4.8 & 9.1 & 11.5 & II.I & \multirow[t]{5}{*}{$<0.0001$} \\
\hline $26-35$ & 9.3 & 12.5 & 13.8 & 13.9 & \\
\hline $36-45$ & 23.7 & 23.8 & 24.9 & 24.5 & \\
\hline $46-55$ & 38.4 & 33.1 & 31.1 & 31.1 & \\
\hline $56-64$ & 23.9 & 21.4 & 18.7 & 19.4 & \\
\hline Mean, years (SD) & $47.2(10.6)$ & $45.1(11.9)$ & $43.7(12.4)$ & $43.8(12.4)$ & $<0.0001$ \\
\hline \multicolumn{6}{|l|}{ Health plan type (\%) } \\
\hline Comprehensive & 11.8 & 10.1 & 9.6 & 10.1 & \multirow[t]{5}{*}{$<0.0001$} \\
\hline HMO & 18.6 & 21.3 & 19.3 & 28.4 & \\
\hline POS & 11.5 & 12.9 & 12.7 & 12.6 & \\
\hline PPO & 56.0 & 53.3 & 55.8 & 46.8 & \\
\hline Other & 2.1 & 2.4 & 2.7 & 2.1 & \\
\hline \multicolumn{6}{|c|}{ Region of residence (\%) } \\
\hline Northeast & 8.8 & 10.1 & 10.2 & 10.9 & \multirow[t]{5}{*}{$<0.0001$} \\
\hline North central & 30.7 & 30.7 & 31.7 & 29.8 & \\
\hline South & 40.8 & 35.9 & 36.8 & 31.2 & \\
\hline West & 19.4 & 22.8 & 20.9 & 27.6 & \\
\hline Unknown & 0.3 & 0.5 & 0.4 & 0.5 & \\
\hline
\end{tabular}

Note: $P$-value based on chi-square test for categorical variable or F-test for continuous variable.

Abbreviations: HMO, health maintenance organization; n, number of patients; POS, point of service; PPO, preferred provider organization; SD, standard deviation; SSRI, selective serotonin reuptake inhibitor.

in VLX-treated patients, 90 days in ECP-treated patients, and 90 days in GSSRI-treated patients. Altogether, DLX-treated patients were less likely to discontinue than patients treated with VLX, ECP, or GSSRIs $(P<0.001)$.

Table 3 presents the multivariate stepwise logistic and Cox proportional hazards regression results for adherence and discontinuation with medication therapy, respectively. ORs and HRs were used to describe the strength of the associations between significant variables and adherence and discontinuation in the final model. Compared with GSSRIs, patients treated with DLX were more likely to be adherent $(\mathrm{OR}=1.66$,

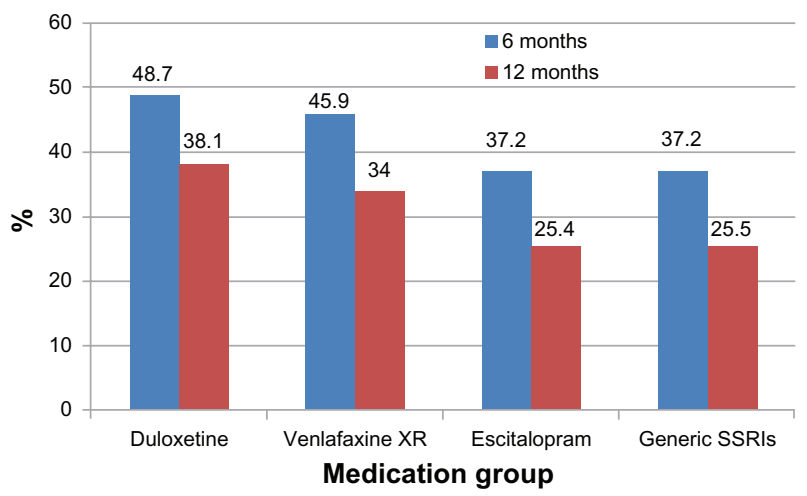

Figure 2 Antidepressant adherence (\%) in patients with major depressive disorder in the six months and twelve months after medication initiation.
CI: $1.57-1.76)$, followed by VLX (OR=1.43, CI: 1.34-1.52). The OR for ECP was 0.99, indicating ECP and GSSRIs were very similar in adherence after adjustment for demographics, comorbid conditions and prior use of medications. Also, patients were more likely to remain adherent if they were older (36 years and above), had comorbid hypersomnia, and had used medications including VLX, ECP, other antidepressants, anticonvulsants, and antimigraine medications in the prior year (Table 3). However, patients were less likely to be adherent if they had comorbid chronic pain diseases (headaches, low back pain, and fibromyalgia), alcohol and

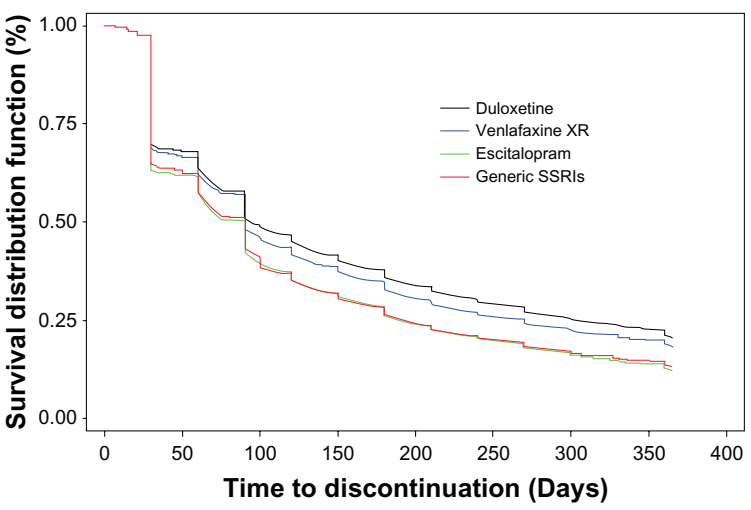

Figure 3 Time to discontinuation with different antidepressant therapy in patients with major depressive disorder in the twelve months after medication initiation. 
Table 3 Multivariate logistic regression and Cox proportional hazards regression: predictors of adherence or persistence with antidepressant therapy

\begin{tabular}{|c|c|c|c|c|}
\hline & \multicolumn{2}{|c|}{ Adherence } & \multicolumn{2}{|c|}{ Discontinuation } \\
\hline & OR & $95 \% \mathrm{Cl}$ & HR & $95 \% \mathrm{Cl}$ \\
\hline \multicolumn{5}{|l|}{ Antidepressant } \\
\hline Generic SSRIs & 1.00 & & 1.00 & \\
\hline Escitalopram & 0.99 & $0.94-1.05$ & 1.02 & $0.997-1.05$ \\
\hline Venlafaxine XR & 1.43 & $1.34-1.52$ & 0.85 & $0.83-0.88$ \\
\hline Duloxetine & 1.66 & I.57-1.76 & 0.81 & $0.78-0.83$ \\
\hline Female gender & 1.05 & $1.00-1.10$ & & \\
\hline \multicolumn{5}{|l|}{ Age (years) } \\
\hline $18-35$ & 1.00 & & 1.00 & \\
\hline $36-45$ & 1.47 & I.38-I.57 & 0.85 & $0.82-0.87$ \\
\hline $46-55$ & 1.65 & I.55-1.75 & 0.80 & $0.78-0.82$ \\
\hline $56-64$ & 2.06 & $1.93-2.21$ & 0.72 & $0.70-0.74$ \\
\hline \multicolumn{5}{|l|}{ Health plan type } \\
\hline PPO & 1.00 & & 1.00 & \\
\hline Comprehensive & 0.91 & $0.84-0.98$ & 1.05 & $1.01-1.09$ \\
\hline HMO & 0.90 & $0.85-0.95$ & 1.02 & $0.99-1.05$ \\
\hline POS & 0.99 & $0.93-1.06$ & 0.99 & $0.92-1.06$ \\
\hline Other & 1.03 & $0.89-1.18$ & 0.95 & $0.92-0.99$ \\
\hline \multicolumn{5}{|c|}{ Geographic region of residence } \\
\hline North central & 1.00 & & 1.00 & \\
\hline Northeast & 1.09 & $1.01-1.18$ & 0.95 & $0.92-0.99$ \\
\hline South & 0.91 & $0.86-0.96$ & 1.06 & $1.04-1.09$ \\
\hline West & 1.05 & $0.98-1.11$ & 0.96 & $0.93-0.99$ \\
\hline \multicolumn{5}{|l|}{ Comorbid diseases } \\
\hline Headaches & 0.89 & $0.84-0.95$ & 1.05 & $1.02-1.09$ \\
\hline \multicolumn{5}{|l|}{ Rheumatoid arthritis } \\
\hline \multicolumn{5}{|l|}{ Osteoarthritis } \\
\hline Low back pain & 0.94 & $0.89-0.99$ & 1.06 & $1.04-1.09$ \\
\hline Fibromyalgia & 0.90 & $0.83-0.95$ & & \\
\hline \multicolumn{5}{|l|}{ Neuropathic pain } \\
\hline \multicolumn{5}{|l|}{ Insomnia } \\
\hline Hypersomnia & 1.23 & $\mathrm{I} .1 \mathrm{I}-\mathrm{I} .36$ & 0.91 & $0.86-0.95$ \\
\hline \multicolumn{5}{|c|}{ Obstructive sleep apnea } \\
\hline \multicolumn{5}{|l|}{ Dysthymic disorder } \\
\hline Anxiety disorders & & & 0.97 & $0.94-0.99$ \\
\hline Alcohol dependence & 0.75 & $0.66-0.85$ & 1.17 & $1.1 \mathrm{I}-1.24$ \\
\hline Drug dependence & 0.66 & $0.56-0.78$ & 1.22 & $|| 4-.|.3|$ \\
\hline \multicolumn{5}{|l|}{ Schizophrenia } \\
\hline \multicolumn{5}{|l|}{ Bipolar disorders } \\
\hline \multicolumn{5}{|l|}{ Organic psychosis } \\
\hline \multicolumn{5}{|c|}{ Diabetes mellitus (except 250.6) } \\
\hline \multicolumn{5}{|c|}{ Hypertensive diseases } \\
\hline \multicolumn{5}{|l|}{ Hepatic diseases } \\
\hline Renal diseases & & & & \\
\hline Prior medications & & & & \\
\hline Hypnotics & & & & \\
\hline Benzodiazepines & & & 1.04 & $1.01-1.06$ \\
\hline Anxiolytics & & & & \\
\hline Other SSRIs & 1.05 & $1.00-1.10$ & & \\
\hline Duloxetine & & & & \\
\hline Venlafaxine & 1.26 & I.18-1.35 & 0.91 & $0.88-0.94$ \\
\hline Escitalopram & 1.13 & $1.07-1.20$ & 0.95 & $0.92-0.96$ \\
\hline TCAs & 1.10 & $1.02-1.19$ & & \\
\hline MAOls & & & & \\
\hline
\end{tabular}


Table 3 (Continued)

\begin{tabular}{|c|c|c|c|c|}
\hline & \multicolumn{2}{|c|}{ Adherence } & \multicolumn{2}{|c|}{ Discontinuation } \\
\hline & OR & $95 \% \mathrm{Cl}$ & HR & $95 \% \mathrm{Cl}$ \\
\hline Other antidepressants & 1.12 & $1.07-1.18$ & 0.94 & $0.92-0.96$ \\
\hline \multicolumn{5}{|l|}{ Typical antipsychotics } \\
\hline \multicolumn{5}{|l|}{ Atypical antipsychotics } \\
\hline \multicolumn{5}{|l|}{ Lithium } \\
\hline Amphetamine & 0.87 & $0.78-0.98$ & I.II & $1.05-1.17$ \\
\hline \multicolumn{5}{|l|}{ Methylphenidate } \\
\hline Modafinil & & & 0.94 & $0.88-0.998$ \\
\hline Anticonvulsants & I.II & $1.05-1.18$ & 0.95 & $0.92-0.98$ \\
\hline Opioids & 0.88 & $0.84-0.92$ & 1.08 & $1.06-1.11$ \\
\hline \multicolumn{5}{|l|}{ NSAIDs } \\
\hline Muscle Relaxant & 0.94 & $0.89-0.99$ & & \\
\hline Antimigraine & 1.16 & $1.07-1.27$ & 0.90 & $0.86-0.94$ \\
\hline
\end{tabular}

Notes: Odds ratio $(\mathrm{OR})$ and its $95 \% \mathrm{Cl}$ for adherence were calculated from a multivariate logistic regression model. Hazard ration (HR) and its $95 \% \mathrm{Cl}$ for discontinuation were calculated from the Cox proportional hazards regression model.

Abbreviations: $\mathrm{Cl}$, confidence interval; HMO, health maintenance organization; MAOls, Monoamine oxidase inhibitors; NSAIDs, Nonsteroidal anti-inflammatory drugs; OR, odds ratio; POS, point of service; PPO, preferred provider organization; SSRI, selective serotonin reuptake inhibitor; TCAs, tricyclic antidepressants.

drug dependence, and prior use of amphetamines, opioids, and muscle relaxants.

As shown in Table 3, compared with GSSRIs, patients treated with DLX were less likely to discontinue medication therapy in the twelve months after initiation $(\mathrm{HR}=0.81$, CI: 0.78-0.83), followed by VLX (HR =0.85, CI: 0.83-0.88). ECP was very similar in the likelihood of discontinuation to GSSRIs (HR = 1.02, CI: 1.00-1.05). Predictors associated with decreased likelihood of discontinuation with antidepressant therapy included older age (36 years and above), hypersomnia, anxiety disorders, and prior use of VLX, ECP, other antidepressants, modafinil, anticonvulsants, and antimigraine medications. Factors associated with increased likelihood of discontinuation were chronic headaches, low back pain, alcohol and drug dependence, and prior use of benzodiazepines, amphetamine, and opioids.

In sensitivity analyses the group differences in adherence and persistence across the four medication cohorts had no essential changes 1) if patients were followed for six months, 2) if an alternative allowable 30-day gap was used to estimate persistence, and 3) if 17-ICD classes of systemic diseases were used to capture comorbid diseases instead of selected comorbid diseases. Similar predictors of adherence and discontinuation were also observed for 6-month and 12-month follow-ups and for an allowable 15-day and 30-day gap.

\section{Discussion}

This study examined adherence and persistence with three branded antidepressants (DLX, VLX, and ECP) and GSSRIs in usual clinical settings. Our results showed that the adherence rate was $46 \%$ for VLX, $49 \%$ for DLX, and $37 \%$ for ECP and GSSRIs in the six months after initiation and declined markedly in the second six months for all antidepressants. Patients treated with DLX were more adherent to and stayed on medication longer than patients treated with VLX, ECP, or GSSRIs. VLX-treated patients had better adherence and longer persistence with therapy than patients treated with ECP or GSSRIs. However, ECP was similar to GSSRIs in adherence and persistence. In addition, multiple demographic and clinical pretreatment factors were associated with treatment adherence and persistence.

The adherence rate was $38.1 \%$ and $34.0 \%$ for DLX and VLX and approximately $25 \%$ for the SSRIs in the year after initiation. About $30 \%$ of patients discontinued their medication within 30 days. The American Psychiatric Association treatment guideline recommends at least four to eight weeks of treatment during the acute phase followed by 16 to 20 weeks of treatment following remission during the continuation phase. ${ }^{40}$ The American College of Physicians guideline recommends treatment for four to nine months after a satisfactory response in patients with a first episode of MDD and an even longer duration of therapy for patients who have had two or more episodes of depression. ${ }^{41}$ Given the consequence of early premature discontinuation and nonadherence, ${ }^{10,33}$ it is important to improve patient adherence to medication therapy and give long enough therapy to reduce relapse and hospitalization and costs.

SNRIs (DLX and VLX) have better adherence and longer persistence than SSRIs including ECP. Over the 1-year study period, the difference in adherence between SNRIs and 
SSRIs was $9 \%$ to $12 \%$ and the difference in length of therapy was 19 to 29 days. There are several possible explanations. First, there were differences in demographic and clinical characteristics of patients initiating SNRIs and SSRIs. SNRItreated patients are more likely to be female, older, and were more complex (recurrent, with more severe and comorbid disorders) than SSRI-treated patients. ${ }^{28}$ These patients may need longer therapy and better adherence. Second, SNRIs may have better effects than SSRIs for severe patients. ${ }^{22,23}$ Third, SNRIs are similar to SSRIs in safety profiles.

Compared with VLX, DLX appears to have better adherence and persistence. The advantage of DLX over VLX may share similar reasons to those for SNRIs over SSRIs and may be attributed to the differences in clinical and pharmacological profiles between the two SNRIs. ${ }^{24,25}$ DLX-treated patients are more complicated and have more comorbid conditions, especially chronic pain diseases, than VLXtreated patients. ${ }^{28,39}$ Compared to VLX, DLX is a relatively balanced SNRI, displaying high affinity for both serotonin and norepinephrine transporters. ${ }^{24}$ In addition to MDD and anxiety, DLX has indications for fibromyalgia and DPNP and has shown efficacy for chronic low back pain. ${ }^{42}$ However, the differences in adherence (4\%) and persistence (nine days) were relatively small, though they were statistically significant. Further research needs to examine if the small differences could be translated into clinically and economically meaningful outcomes.

ECP is the only branded SSRI in the United States. Our results show that ECP has very similar adherence and persistence profiles compared to GSSRIs. This finding is not surprising because ECP is an SSRI and has similar clinical and pharmacological profiles compared to other SSRIs ${ }^{43}$ and because ECP-treated patients are also very similar to GSSRI-treated patients in demographics and comorbid conditions. $^{28}$

Consistent with previous studies, ${ }^{13-18,20,21}$ this study found that multiple demographical and clinical factors were associated with adherence and persistence. For example, older patients were more likely to be adherent with antidepressant therapy. Eaddy and colleagues ${ }^{12}$ studied the association between SSRI utilization patterns and use of health care services and reported that patients who discontinued therapy in the first 90 days tended to be younger. Certain comorbid diseases can increase or decrease the odds of discontinuation of antidepressant therapy. Patients with chronic headaches, low back pain, and alcohol and drug dependence were more likely to discontinue therapy, while patients with hypersomnia were more likely to remain adherent. Also, the prior use of certain medications can affect adherence and persistence. Patients who have been prescribed VLX, ECP, anticonvulsants, and antimigraine medications have decreased odds of discontinuation, while patients with prior use of benzodiazepines, amphetamine, and opioids have increased odds of discontinuation. It would be warranted to investigate why certain comorbid disorders and medications are related to poor adherence and early discontinuation with antidepressant therapy.

\section{Limitations}

Several limitations must be considered in this retrospective study using a claims database. Claims database analyses have potential selection bias, miscoding of information and consequent biases in estimation. Also, adherence and persistence were estimated based on the presence of a claim for a filled prescription rather than real consumption of the medication as reported by patients or assessed by objective measures. Other limitations of this study include: the lack of detailed clinical data on the severity of depression, illness history, duration of current episode, and patient responses to antidepressants; and the lack of detailed social demographic data on education, ethnicity, employment status, income, and family environment; and the absence of information about physician and patient belief and preference. Demographic and clinical differences were observed between the cohorts of patients initiating SNRIs and SSRIs. While attempts were made to control for these baseline differences in measured variables through regression analysis, potential differences in the above unmeasured variables are unaccounted for - except in as much as they are correlated with measured variables. Prospective observational studies are needed to examine whether these findings can be generalized to the real-world clinical setting. In addition, although adherence and persistence were statistically different across study medications, further research is needed to examine whether the statistical differences can be translated into clinically and economically meaningful consequences.

\section{Conclusion}

The adherence rate was $34 \%$ to $38 \%$ for SNRIs and approximately $25 \%$ for SSRIs in the year after initiation. About $30 \%$ of patients discontinued their medication within 30 days. DLX and VLX treated patients had better adherence and longer length of therapy with the medication than patients treated with ECP or GSSRIs. While DLX treated patients had statistically significantly better adherence and persistence than VLX, the observed differences were 
relatively small and further research is needed to examine if these differences translate into clinically and economically meaningful outcomes. Adherence and persistence between ECP and GSSRIs were similar. In addition, multiple demographic and clinical factors are associated with adherence and persistence. Given the clinical and economic benefits of better adherence and persistence with antidepressant therapy, targeting interventions to patients who are at high risk for nonadherence and early discontinuation of therapy should be considered an important part of medication therapy for major depression.

\section{Acknowledgments}

The authors thank Dr Jarrett Coffindaffer and Ms Teri Tucker of i3 Statprobe for their assistance in preparing this manuscript. This study was funded by Eli Lilly and Company.

\section{Disclosure}

$\mathrm{X}$ Liu is a former employee, while Y Chen and D Faries are current employees of Eli Lilly and Company.

\section{References}

1. Kessler RC, Chiu WT, Demler O, Merikangas KR, Walters EE, Prevalence, severity, and comorbidity of 12-month DSM-IV disorders in the National Comorbidity Survey Replication. Arch Gen Psychiatry. 2005;62:617-627.

2. World Health Organization. The World Health Report 2004: Changing History, Annex Table 3: Burden of disease in DALYs by cause, sex, and mortality stratum in WHO regions, estimates for 2002. World Health Organization. Geneva, Switzerland; 2004.

3. Greenberg PE, Kessler RC, Birnbaum HG, et al. The economic burden of depression in the United States: how did it change between 1990 and 2000? J Clin Psychiatry. 2003;64:1465-1475.

4. Stafford RS, MacDonald EA, Finkelstein SN. National Patterns of Medication Treatment for Depression, 1987 to 2001. Prim Care Companion J Clin Psychiatry. 2001;3:232-235.

5. Caley CF, Kando JC. SSRI efficacy-finding the right dose. J Psychiatr Pract. 2002;8:33-40

6. Cantrell CR, Eaddy MT, Shah MB, Regan TS, Sokol MC. Methods for evaluating patient adherence to antidepressant therapy: a real-world comparison of adherence and economic outcomes. Med Care. 2006; 44:300-303.

7. Cramer JA, Roy A, Burrell A, et al. Medication compliance and persistence: terminology and definition. Value Health. 2008;11:44-47.

8. Ruhé HG, Huyser J, Swinkels JA, Schene AH. Switching antidepressants after a first selective serotonin reuptake inhibitor in major depressive disorder: a systematic review. J Clin Psychiatry. 2006;67:1836-1855.

9. Khandker RK, Kruzikas DT, McLaughlin TP. Pharmacy and medical costs associated with switching between venlafaxine and SSRI antidepressant therapy for the treatment of major depressive disorder. J Manag Care Pharm. 2008;14:426-441.

10. Melfi CA, Chawla AJ, Croghan TW, Hanna MP, Kennedy S, Sredl K. The effects of adherence to antidepressant treatment guidelines on relapse and recurrence of depression. Arch Gen Psychiatry. 1998;55: $1128-1132$.

11. Lin EH, Von Korff M, Bush T, Simon GE, Walker E, Robinson P. The role of the primary care physician in patients' adherence to antidepressant therapy. Med Care. 1995;33:67-74.
12. Eaddy MT, Druss BG, Sarnes MW, Regan TS, Frankum LE. Relationship of total health care charges to selective serotonin reuptake inhibitor utilization patterns including the length of antidepressant therapy: results from a managed care administrative claims database. J Manag Care Pharm. 2005;11:145-150.

13. Nantz E, Liu-Seifert H, Skljarevski V. Predictors of premature discontinuation of treatment in multiple disease states. Patient Prefer Adherence. 2009;3:31-43.

14. Bosworth HB, Voils CI, Potter GG, Steffens DC. The effects of antidepressant medication adherence as well as psychosocial and clinical factors on depression outcome among older adults. Int $J$ Geriatr Psychiatry. 2008;3:129-134.

15. Bulloch AG, Patten SB. Non-adherence with psychotropic medications in the general population. Soc Psychiatry Psychiatr Epidemiol. 2010; 45:47-56.

16. Demyttenaere K, Enzlin P, Dewe W, et al. Compliance with antidepressants in a primary care setting, 2: the influence of gender and type of impairment. J Clin Psychiatry. 2001;62 Suppl 22:S34-S37.

17. Demyttenaere K, Bonnewyn A, Bruffaerts R, et al. Clinical factors influencing the prescription of antidepressants and benzodiazepines: results from the European study of the epidemiology of mental disorders (ESEMeD). J Affect Disord. 2008;110:84-93.

18. Lin EH, Von Korff M, Ludman EJ, et al. Enhancing adherence to prevent depression relapse in primary care. Gen Hosp Psychiatry. 2003;25: 303-310.

19. Claxton AJ, Cramer J, Pierce C. A systematic review of the associations between dose regimens and medication compliance. Clin Ther. 2001;23: 1296-1310.

20. Pompili M, Serafini G, Del Casale A, et al. Improving adherence in mood disorders: the struggle against relapse, recurrence and suicide risk. Expert Rev Neurother. 2009;9:985-1004.

21. Warden D, Trivedi MH, Wisniewski SR, et al. Predictors of attrition during initial (citalopram) treatment for depression: a STAR*D report. Am J Psychiatry. 2007;164:1189-1197.

22. Nelson JC, Mazure CM, Jatlow PI, Bowers MB Jr, Price LH. Combining norepinephrine and serotonin reuptake inhibition mechanisms for treatment of depression: a double-blind, randomized study. Biol Psychiatry. 2004;55:296-300.

23. Papakostas GI, Thase ME, Fava M, Nelson JC, Shelton RC. Are antidepressant drugs that combine serotonergic and noradrenergic mechanisms of action more effective than the selective serotonin reuptake inhibitors in treating major depressive disorder? A meta-analysis of studies of newer agents. Biol Psychiatry. 2007;62:1217-1227.

24. Beique JC, Lavoie N, de Montigny C, Debonnel G. Affinities of venlafaxine and various reuptake inhibitors for the serotonin and norepinephrine transporters. Eur J Pharmacol. 1998;349:129-132.

25. Cipriani A, Furukawa TA, Salanti G, et al. Comparative efficacy and acceptability of 12 new-generation antidepressants: a multipletreatments meta-analysis. Lancet. 2009;373:746-758.

26. Beique JC, de Montigny C, Blier P, Debonnel G. Venlafaxine: discrepancy between in vivo 5-HT and NE reuptake blockade and affinity for reuptake sites. Synapse. 1999;32:198-211.

27. Duloxetine hydrochloride. Prescribing information. Indianapolis, IN: Lilly USA LLC; 2009.

28. Liu, XC, Chen Y, Faries D, Miner C, Swindle R. Impact of comorbidities on antidepressant initiation: duloxetine, venlafaxine, and escitalopram versus other SSRIs. 38th American College of Clinical Pharmacology Annual Meeting; 2009 Sept 13-15; San Antonio, TX.

29. Crown WH, Finkelstein S, Berndt ER, Ling, et al. The impact of treatment-resistant depression on health care utilization and costs. J Clin Psychiatry. 2002;63:963-971.

30. Matza LS, Rajagopalan KS, Thompson CL, de Lissovoy G. Misdiagnosed patients with bipolar disorder: comorbidities, treatment patterns, and direct treatment costs. J Clin Psychiatry. 2005;66:1432-1440.

31. Iosifescu DV, Bankier B, Fava M. Impact of medical comorbid disease on antidepressant treatment of major depressive disorder. Curr Psychiatry Rep. 2004;6:193-201. 
32. Iosifescu DV, Clementi-Craven N, Fraguas R, et al. Cardiovascular risk factors may moderate pharmacological treatment effects in major depressive disorder. Psychosom Med. 2005;67:703-706.

33. Liu XC, Ye W, Watson P, Tepper P. Use of benzodiazepines, hypnotics, and anxiolytics in major depressive disorder: association with chronic pain diseases. J Nerv Ment Dis. 2010;198:544-550.

34. Ong JC, Gress JL, San Pedro-Salcedo MG, Manber R. Frequency and predictors of obstructive sleep apnea among individuals with major depressive disorder and insomnia. J Psychosom Res. 2009;67: 135-141.

35. Shelton C. Factors impacting the selection of antidepressant treatment in patients with major depressive disorder at risk for nonadherence. CNS Spectr. 2009;14:15-19.

36. Vaccarino AL, Sills TL, Evans KR, Kalali AH. Multiple pain complaints in patients with major depressive disorder. Psychosom Med. 2009;71: $159-162$.

37. Wu EQ, Greenberg PE, Yang E, Yu AP, Ben-Hamadi R, Erder MH. Treatment persistence, healthcare utilisation and costs in adult patients with major depressive disorder: a comparison between escitalopram and other SSRI/SNRIs. J Med Econ. 2009;12:124-135.

38. Kroenke K, Krebs EE, Bair MJ. Pharmacotherapy of chronic pain: a synthesis of recommendations from systematic reviews. Gen Hosp Psychiatry. 2009;31:206-219.
39. Ye WY, Zhao Y, Robinson R, Swindle R. Predictors of treatment initiation with duloxetine compared with venlafaxine $\mathrm{XR}$ among patients with major depressive disorder in US managed-care setting. 13th International Meeting of the International Society for Pharmacoeconomics and Outcomes Research; 2008 May 3-7; Toronto, ON.

40. Karasu TB, Gelenberg A, Merriam AE, Wang P. American Psychiatric Association practice guideline for the treatment of patients with major depressive disorder. Am J Psychiatry. 2000;157:1-45.

41. Qaseem A, Snow V, Denberg TD, Forciea MA, Owens DK. Using second-generation antidepressants to treat depressive disorders: a clinical practice guideline from the American College of Physicians. Ann Intern Med. 2008;149:725-733.

42. Skljarevski V, Zhang S, Chappell AS, Walker DJ, Murray I, Backonja M. Maintenance of effect of duloxetine in patients with chronic low back pain: a 41-week uncontrolled, dose-blinded study. Pain Med. 2010;11:648-657.

43. Gartlehner G, Gaynes BN, Hansen RA, et al. Comparative benefits and harms of second-generation antidepressants: background paper for the American College of Physicians. Ann Intern Med. 2008;149: 734-750.
ClinicoEconomics and Outcomes Research

\section{Publish your work in this journal}

ClinicoEconomics \& Outcomes Research is an international, peerreviewed open-access journal focusing on Health Technology Assessment, Pharmacoeconomics and Outcomes Research in the areas of diagnosis, medical devices, and clinical, surgical and pharmacological intervention. The economic impact of health policy and health systems

\section{Dovepress}

organization also constitute important areas of coverage. The manuscript management system is completely online and includes a very quick and fair peer-review system, which is all easy to use. Visit http://www.dovepress.com/testimonials.php to read real quotes from published authors.

Submit your manuscript here: http://www.dovepress.com/clinicoeconomics-and-outcomes-research-journal 